\title{
A COMPARISON OF STRUCTURED AND UNSTRUCTURED NAVIGATION THROUGH A CBT PACKAGE
}

\section{N. A. STANTON and R. B. STAMMERS}

\author{
Applied Psychology Division. Aston University, Birmingham B4 7ET. England
}

\begin{abstract}
The advent of hypertext has opened up new possibilities in computer-based training. The design of courseware without any predetermined structure could make the designer's task easier, and allow greater flexibility for the trainee to structure the learning environment to suit their own learning style. This investigation was concerned with the exploration of performance differences in structured and unstructured training environments. In the structured condition, subjects encountered presequenced training and practice modules. For the unstructured condition, subjects determined their own sequence of modules. It was proposed that performance may be better in the unstructured condition. The findings indicate that this depends upon individual differences in cognitive style, some styles seemingly better at exploiting the unstructured learning environment than others.
\end{abstract}

\section{INTRODUCTION}

The virtues of the computer as an instructional medium are held to be benefits such as; learner pacing of instruction, the interactive quality of the medium, immediate feedback, and the individualization of the learning environment [1-3]. It is the interactive and individualized nature of computer-based training (CBT) that sets it apart from other instructional media. The effectiveness of any CBT package is totally dependent upon its design [4, 5]. This has led to theories of instructional design, and the development of design procedures for CBT. Instructional design theories largely remain to be tested empirically, and none of the theories proposed to date have proved to be universally adequate.

This shortcoming may no longer be of concern as a new dimension is presented with the advent of technology that is able to realize the hypertext philosophy [6]. Hypertext is the idea that knowledge (be it in the form of text or graphics) may be linked in many ways, providing no formal structure, allowing the individual to explore the knowledge domain at will. The hypertext approach has certain advantages over structured training. For example, courseware authors do not have to concern themselves with structuring the information in any particular order. Trainees may approach the learning environment with a wealth of previous experience which can enable them to be directive in their own learning. The existence of learning skills and strategies may transfer to the new environment [7]. If learners are able to choose how the information and instruction is presented to them, in the manner that best suits their own style of learning, then, the whole process may be more efficient. In addition, this phenomenon offers the potential for speeding up the process of authoring course materials. There are two possible ways in which they may occur. Firstly, the author does not have to pre-sequence the instruction, as this is done by the trainee at the time of training. Secondly, existing courseware can be revised, updated, and added to, without interfering with other parts of the courseware, making the process quicker and easier.

This investigation was concerned with a comparison of a training environment that presented the instruction modules in a structured format over which the trainee had no influence, with another in which the trainee was able to determine the sequence of the instructional and practice phases freely. Under examination was the question of trainees' performance, both within the training environment and subsequently on the task. It was 
postulated that the unstructured condition could lead to greater efficiency in training, without any decrement in transfer of task performance.

Another issue was the trainees' ability to manage the unstructured environment, which was considered to be related to their style of learning. Cognitive styles were, therefore, also to be investigated. Several cognitive styles in learning have been postulated (e.g. review by Robertson [8]). Pask [9] identified the difference between a "serialist" (a stepby-step approach in a linear fashion, increasing understanding by small increments) and a "holist" (a more global approach, involving testing assumptions of the overall structure of the task). The hierarchical structure of the task under investigation was such that two further styles might emerge. These are a "top down" strategy (starting with higher-order procedures, moving down to lower-order procedures) and a "bottomup" strategy (starting with lower-order procedures, moving up to higher-order procedures). The issue of self assessment was also investigated, but is not under consideration in this paper.

\section{METHOD}

\section{Subjects}

Sixty subjects were employed for the full investigation. Twenty in a structured self assessment condition, 20 in a structured condition without self assessment and 20 in the unstructured condition. Only the results of the latter two groups are considered here. All the subjects were undergraduates at Aston University, and the groups were matched for age and sex. Subjects were alone in laboratory cubicles during their participation in the investigation.

\section{Design}

The task involved interacting with a simplified "process control" plant. It required the subjects to monitor the plant status, to respond to alarms, and to locate and reset faults through a set of hierarchically organized plant indicators. The training modules were:

\section{Orientation:}

\section{Principles: \\ Screen: \\ Keyboard: Monitor Output: Respond to Alarm: Delete Key: Locus of Fault:}

Gathers subject's data (age, sex), shows subject how to use the mouse to move objects on the screen, orientates the subject to the learning objectives, and demonstrates the principle of an information hierarchy.

Demonstrates the hierarchical nature of the indicators and processes. Explains the functional area of the screen.

Shows the functions of the keys.

Demonstrates how to monitor the overall system state.

Demonstrates how to respond in the event of an alarm.

Demonstrates how to use the delete key.

Demonstrates how to locate a fault through the hierarchy of indicators and reset the process.

Each of the training modules had a corresponding practice module, which in the case of Locus of Fault used an interactive simulator.

Both the structured and unstructured groups' training contained all of these modules, the difference being that the second group could select the order of presentation, and choose not to view any of the modules. The unstructured group selected the modules to be viewed via an overview screen. They returned to this after they had finished a particular module to select the next. Both groups could repeat modules as required. The structured group were presented with each of the modules in the order listed.

Development of the CBT package took in the region of $300 \mathrm{~h}$, which included learning the functionality of Coursebuilderтм. This authoring package was particularly good for presenting animated graphics and making the environment interactive, which maximized the positive features of CBT. However, it was not a good medium for producing a simulator, which took a great deal of time to develop. It was difficult to judge if the 
structured condition took longer to develop than the unstructured condition. This was due to fact that both conditions were developed together. The structured condition required considerable pre-development effort in task analysis and structuring. Whilst the unstructured condition benefited from this, it could have been developed during the authoring, requiring less rigorous pre-development planning.

\section{Procedure}

The procedure for the investigation was as follows:

(I) Subjects were given a demonstration of how to use the pointing device.

(2) The subjects were assigned to either of the experimental conditions depending on age and sex

(3) Subjects then answered a computer-based self confidence questionnaire.

(4) The training began.

(5) When subjects called the experimenter to notify that the training had finished, they were then moved to another cubicle, and asked to control the "plant" (the transfer session).

(6) Subjects were asked to complete a questionnaire.

(7) One week later subjects returned to run the task again (the retention session).

(8) Subjects in the unstructured condition were measured on the Embedded Figures Test.

(9) Subjects were paid £5.00.

\section{Equipment}

The training was presented on a Macintosh subjects interacted with the material using a mouse. The training was written in Coursebuilderтм. The simulated task was presented on a BBC Model B Microcomputer, with a 6502 Second Processor, via a colour monitor and a purpose-built keyboard.

\section{Measurement}

Measurement was taken of the time spent in each of the training and practice modules, performance in the practice modules, the order of each of the modules, the transfer and retention performance, a post-test questionnaire, and individual difference measures of subjects in the unstructured group using the Embedded Figures Test.

\section{Analysis}

The time and performance data were analysed using ANOVA. This was to test for differences between the time spent by subjects in the structured and unstructured conditions in the training and practice modules. The transfer and retention performance data was also analysed in this way. A nonparametric technique (the Mann-Whitney utest) was employed to investigate the differences between the two conditions in terms of the number of modules viewed.

\section{RESULTS}

The results showed that: there was no significant difference in the training time between the two conditions (structured and unstructured training modules). However, the difference between the transfer and retention performance between the two conditions approaches significance $\left(F_{1.38}=2.788, P<0.1\right)$ with better operational performance in the unstructured condition. Both groups performance improved significantly from the transfer task to the retention task (Unstructured condition $\mathrm{F}_{1.38}=11.258, P<0.01$; Structured condition $\left.\mathrm{F}_{1.38}=6.468, P<0.05\right)$. As Fig. I illustrates, a significant difference was found between the variation of the number of modules viewed in the two conditions (Mann-Whitney $U, P<0.05$ ). Some subjects in the unstructured condition explored more of some modules whilst others explored considerably less. Some subjects in the unstructured condition also repeated significantly more training and practice modules 
(Mann-Whitney $U, P<0.05$ ). The number of modules viewed was then related to the subjects' reported learning style in the unstructured condition. Subjects were classified according to their reported learning style, for example; "I looked at the most important things first" (top down), "I progressed from more basic information upwards" (bottom up), and "I went through the modules in an anticlockwise sequence from the overview screen" (serial). Statistical significance was demonstrated between a top down style $(\mathrm{N}=7)$ and a bottom up style $(\mathrm{N}=7)$ on the number of modules viewed (Mann-Whitney $\mathrm{U}, P<0.01)$.

\section{DISCUSSION}

The results suggest that although there was no significant difference in the training time between the conditions, the unstructured environment may lead to an improved task performance. This is probably because it allowed individuals to utilize the unstructured training environment in a manner that suits their own particular learning style. Trainees may actually be able to process information more effectively if it is presented in a manner that is closely matched to their cognitive style [10].

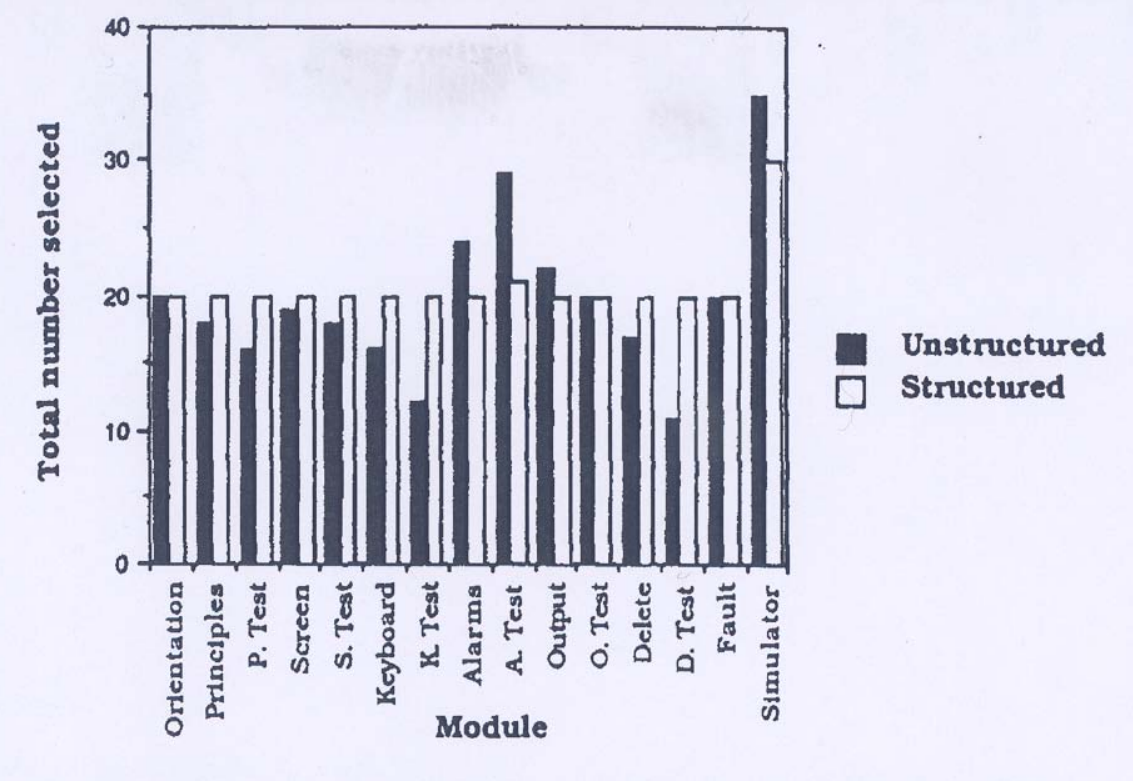

Fig.1. Total modules selected in the structured and unstructured groups.

This is further supported by the finding that although some subjects in the unstructured condition go through less training and practice modules, some subjects repeated more. This may be due to the subject being more involved in a directive learning mode, therefore actively testing assumptions and seeking information, rather than passively waiting for the information to be presented. This behaviour may also provide an explanation of why there was no significant difference in the training time between the two conditions, as some subjects in the unstructured condition may have spent more time in the modules that they considered important to equip them with the necessary skills to function at optimum performance.

Based upon self report, three broad band classifications of; top down, bottom up and serialist evolved. Subjects in the top down group explored fewer modules than those in the bottom up group. This is not surprising given that working top down would allow subjects to make inferences about the lower modules, and therefore not have to view them. The results further suggest that this environment does not suit everyone.

Hartley [11] acknowledges whilst it may be ideal to encourage trainees to select the information in a manner that best suits their own learning styles, it is possible that their 
freestyle is inappropriate in a particular instructional environment. A dislike of the lack of structure was reported by some subjects, concurring with the suggestion that the freedom allowed by hypermedia may be too much for some trainees and some guidance may be necessary [12]. In addition, non-sequential presentation may be inappropriate for learning procedural skills. Further investigations should have the emphasis upon advice rather than control, as too much structure may make the environment inflexible and tedious. The subjects' ability to manage the unstructured training may be dependent upon how clearly they can relate the task goals to the learning environment.

It is probably fair to suggest that the unstructured condition may be a better training environment for some individuals, whilst others may do better in the structured condition. This is most likely to be dependent on the individual's own learning style and abilities. Therefore, the successful management of the environment may be related to these factors. Finally, it should be noted that the unstructured condition presented in this investigation was not true hypertext. The subjects were able to navigate between modules, but they were not able to do this from within the training and practice modules.

In conclusion, performance may be improved in the unstructured training environment, but this is likely to be dependent upon the ability of the subjects to organize a suitable learning environment of their own. Hartley [11] suggests that this would require the trainee to accept greater responsibility for their own learning, and that this may be related to a preferred style of interaction.

Acknowledgement -The authors would like to thank their colleagues, Michael Carey and Ray Taylor for their advice and assistance throughout this investigation.

\section{REFERENCES}

1. Hudson W. J., Computer-based training: will it replace you? CT/M: The Human Element 15, 13-21 (1982).

2. Hobson P. G., Microcomputers in technical training: the Micro TICCIT system.

Telecommunications for Pacific Development. PTC'85. Toward a Digital World, pp. 380-383. Honolulu, Hawaii (1985).

3. Guest M. R., Training psychology and technical based training. In Technical Based Training (Edited by Labinger M. and Finch P. J.). Pergamon Infotech, London (1986).

4. Mahoney F. X. and Lyday N. L., Design is what counts in computer-based training. Train. Dec. J. 38, 4041 (1984).

5. Ashcroft R. A. M., CAL in Industry-the next years. Proceedings of the Fifth Canadian

Symposium on Industrial Technology, pp. 185 -189 (1986).

6. Nelson T., The call of the ocean: Hypertext universal and open. Hyperage May -June, 5 -7 (1988).

7. Perry P. and Downs S., Skills, strategies and ways of learning: can we help people learn how to learn? Progm. Learn. educ. Technol. 22, 177-181 (1985).

8. Robertson I. T., Individual differences in information processing strategy and style.

Proceedings of the International Conference on Man/Machine Systems, pp. 85-88. IEE, London (1982).

9. Pask G., Strategies and styles of learning. Br. J. educ. Psychol. 46, 128 -148 (1976).

10. Brooks L. W., Simutis Z. M. and O'Neil H. F., The role of individual differences in learning strategies. In Individual Differences in Cognition (Edited by Dillon R. F.). Vol. 2. Academic Press, New York (1985).

11. Hartley J. R., Some psychological aspects of computer-assisted learning and teaching.

Progm. Learn. educ. Technol. 22, 140 - 149 (1985).

12. Hammond N. and Allinson L., (1988) Taking the hype out of hypertext: why it's inadequate for learning. Proceedings of the 4th European Conference on Cognitive Ergonomics (Edited by Green T. R. G.). Cambridge, England (1988). 